\title{
ESTUDO DA APLICAÇÃO DE ESTAÇÕES TOTAIS PARA A DETERMINAÇÃO DE DESLOCAMENTOS RELATIVOS EM LABORATÓRIO
}

\author{
Application of total stations for the determination of relative displacements in \\ laboratory. \\ PEDRO LUIS FAGGION ${ }^{1}$ \\ LUIS AUGUSTO KOENIG VEIGA ${ }^{1}$ \\ TARCISIO FERREIRA SILVA ${ }^{2}$ \\ SILVIO HENRIQUE DELLESPOSTE ANDOLFATO ${ }^{3}$ \\ ${ }^{1}$ Universidade Federal do Paraná \\ ${ }^{2}$ Universidade Federal de Pernambuco \\ ${ }^{3}$ Universidade Tecnológica Federal do Paraná - Campus Pato Branco \\ faggion@ufpr.br,kngveiga@ufpr.br, tarcisiofs@yahoo.de, andolfato@utfpr.edu.br
}

\section{RESUMO}

O posicionamento de precisão tem sido um desafio para atender as necessidades dos desenvolvimentos atuais, como é o caso das locações industriais que podem requerer precisões milimétricas ou melhores no posicionamento de equipamentos e também apresentam limitações relacionadas ao espaço onde os trabalhos são executados, dificultando a realização dos levantamentos. Os equipamentos tradicionalmente empregados são ditos de alta precisão (leituras angulares com precisão de um segundo ou melhor, e linear que podem ser superior a $\pm(1 \mathrm{~mm}+$ $1 \mathrm{ppm})$ ). O posicionamento absoluto com estações totais estará limitado ás precisões nominais para a medição das direções e distâncias, assim buscou-se com este estudo de caso avaliar a qualidade da determinação relativa de deslocamentos (distâncias) de pontos em situações que simulem um ambiente industrial, onde existem limitações no posicionamento dos instrumentos durante a determinação das medições. Neste estudo, foi montada uma estrutura móvel sobre os trilhos de um interferômetro instalado no Laboratório de Instrumentação Geodésica da Universidade Federal do Paraná, o qual permite determinar deslocamentos com precisão de $0,01 \mathrm{~mm}$ através de medidas com laser, e nesta estrutura foram instalados prismas refletores para que fosse possível determinar os deslocamentos empregando-se estações totais. Foram utilizados 4 modelos de estações totais, com 
diferentes precisões. Os testes foram realizados em laboratório, com distâncias entre os pontos monitorados a posição onde foram instaladas as estações totais inferiores a 6 metros. Os resultados obtidos mostram que foi possível, para este estudo, determinar deslocamentos com acurácia melhor do que um milímetro, dependendo do modelo de equipamento utilizado.

Palavras-chave: Posicionamento de Precisão; Topografia Industrial.

\section{ABSTRACT}

The accurate positioning has been a challenge to meet the needs of current developments, as in the case of industrial locations that may require sub-millimeter precision in the positioning of equipments and also have limitations related to the area where the work will be performed, making the surveying difficult in some cases. The surveying equipment used in this situation is traditionally classified as high precision ones (angle measurement accuracy of a second and distance measurement accuracy of $\pm(1 \mathrm{~mm}+1 \mathrm{ppm})$ or better). The positioning accuracy with total station is limited to the nominal accuracy of the instruments (both angle and distance). In this work the quality of relative displacements measurements of points in situations that simulate an industrial environment was evaluated, where there is limitation in the instrument set up process. The tests were performed in the laboratory, with distances between points and the total station fewer than 5 meters. Absolute positioning with total stations will be limited by the nominal precision of directions and distances, so in this research it was tried to evaluate the quality of measurement of relative displacements (distances) of points in situations that simulate an industrial environment where there is limitation in the setup of instruments for the determination of the measurements. In this paper was used a movable structure mounted on the rails of an interferometer. This allows the deformation of displacements to within $0.01 \mathrm{~mm}$ by using laser measurements. In this structure a prism reflector was fixed, making possible to determine the displacements employing stations total. Four models of total stations, with different accuracies were used. The tests were performed in the laboratory, with distances between the points monitored and the position of the total stations fewer than 6 meters. The results showed that it is possible, for this study, to determine displacements with accuracy better than a millimeter, depending on the equipment model used.

Keywords: Precision positioning; Industrial Surveying.

\section{INTRODUÇÃO}

Em diversas aplicações, mas notadamente na área de controle e monitoramento de grandes estruturas antrópicas e locações industriais, existe interesse na determinação da posição de pontos em diferentes épocas para o acompanhamento de possíveis deslocamentos dos mesmos. Estas informações são importantes para o estudo da estrutura e para o gerenciamento de questões de segurança. 
Tradicionalmente são empregados em ambientes industriais equipamentos como teodolitos de alta precisão (com precisão igual ou melhor que um segundo de arco) e miras horizontais de invar para a determinação de distâncias. Existem também alguns modelos de estações totais que incorporam sistemas de medição de alta precisão (como precisão angular melhor que 1" e linear que podem ser superior $\mathrm{a} \pm(1 \mathrm{~mm}+1 \mathrm{ppm}))$.

O posicionamento absoluto com estações totais estará limitado ás precisões nominais para a medição das direções horizontais, ângulos zenitais e distâncias inclinadas, assim buscou-se com este estudo de caso avaliar a qualidade da determinação relativa do deslocamento de pontos em situações que simulem um ambiente industrial, onde existem limitações no posicionamento dos instrumentos durante as medições. Neste experimento, os testes forma realizados em laboratório, com distâncias entre a estação total e pontos monitorados inferiores a 5 metros. .

Para validação e comparação dos resultados obtidos, os deslocamentos também foram determinados empregando-se o interferômetro laser, que permite determinar deslocamentos com precisão sub-milimétrica. Devido a sua precisão, este foi adotado como padrão de referência ${ }^{1}$.

\section{METODOLOGIA}

O experimento foi realizado nas dependências do Laboratório de Instrumentação Geodésica da Universidade Federal do Paraná (LaIG). Neste laboratório está disponível um sistema para a calibração de miras verticais de invar composto por um conjunto de trilhos e um interferômetro laser da marca Hewlett Packard modelo 5508A. Na atual configuração deste sistema é possível determinar distâncias com precisão de $0,01 \mathrm{~mm}$. Desta forma será possível comparar este deslocamento com os obtidos empregando-se as estações totais, avaliando a acuracidade na determinação relacionada, uma vez que dispõe-se de um valor de referência para comparação.

Sobre os trilhos do interferômetro é possível mover um carrinho controlado por um motor, e os deslocamentos realizados (distâncias percorridas em relação a uma origem) são controlados através do sistema de medida a laser. Sobre este carrinho foi montada uma plataforma onde é instalado o prisma refletor (figura 1)

Os modelos de estações totais testado, bem como suas precisões nominais são apresentados na Tabela 1 .

\footnotetext{
${ }^{1}$ Padrão de referência: padrão, geralmente tendo a mais alta qualidade metrológica disponível em um dado local ou em uma dada organização a partir do qual as medições lá executadas são derivadas (FAGGION, 2001)
}

Bol. Ciênc. Geod., sec. Artigos, Curitiba, v. 19, nº 1, p.34-44, jan-mar, 2013. 
Tabela 1 - Estações Totais empregadas no experimento.

\begin{tabular}{c|c|c|c}
\hline Modelo & $\begin{array}{c}\text { Precisão } \\
\text { Linear }\end{array}$ & $\begin{array}{c}\text { Precisão } \\
\text { Angular }\end{array}$ & $\begin{array}{c}\text { Classificação de acordo } \\
\text { com a NBR 13.133 }\end{array}$ \\
\hline Leica TC 2003 & $\pm(1 \mathrm{~mm}+1 \mathrm{ppm})$ & $1{ }^{\prime}$ & Precisão Alta \\
\hline Zeiss Elta S20 & $\pm(2 \mathrm{~mm}+2 \mathrm{ppm})$ & $3{ }^{\prime}$ & Precisão Média \\
\hline Leica TC 407 & $\pm(5 \mathrm{~mm}+2 \mathrm{ppm})$ & $7{ }^{\prime \prime}$ & Precisão Média \\
\hline Topcon GTS239W & $\pm(3 \mathrm{~mm}+3 \mathrm{ppm})$ & $9{ }^{\prime}$ & Precisão Baixa \\
\hline
\end{tabular}

* Precisões indicadas nos manuais dos equipamentos

Figura 1 - Disposição dos equipamentos utilizados.

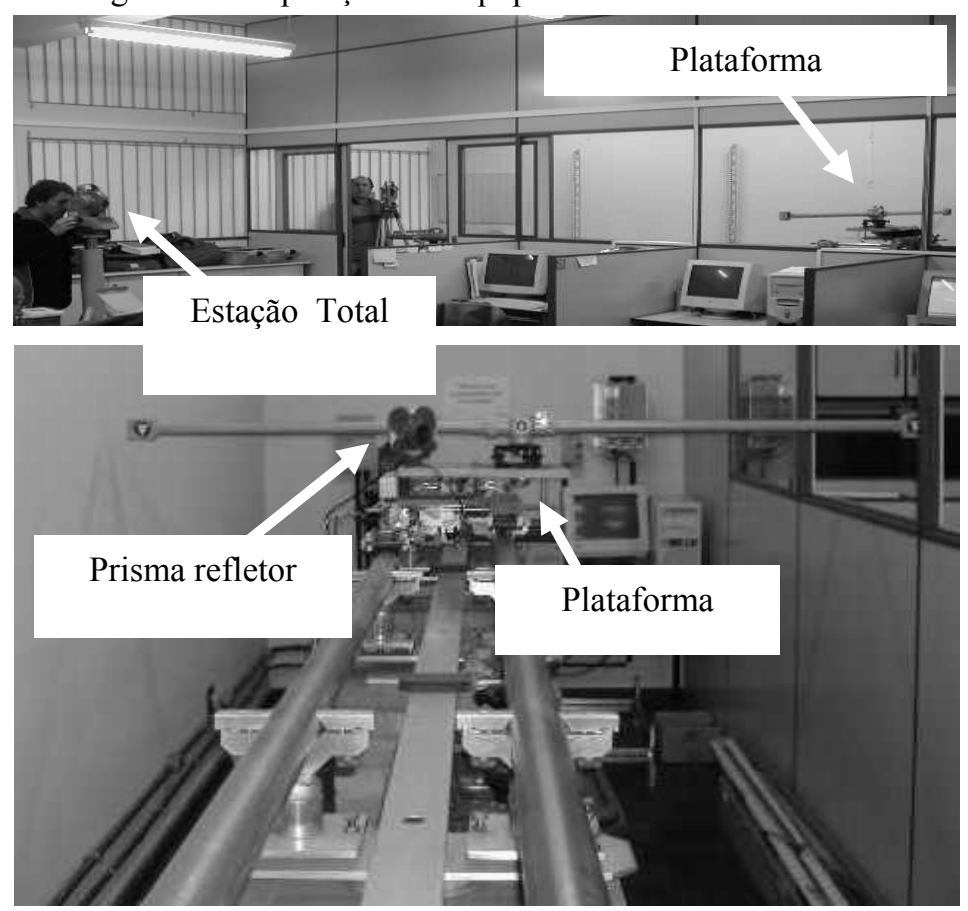

Além disto, foi utilizado um prisma Leica modelo GPR 111 (para as estações Leica e Zeiss) e um prisma Topcon, compatível com a respectiva estação testada.

Salienta-se que todos os experimentos foram realizados em ambiente laboratorial, com temperatura controlada. Os vidros que isolam a sala do interferômetro foram retirados de forma a não inviabilizar as leituras efetuadas nos primas. As estações foram estacionadas sobre um tripé industrial que garante a estabilidade durante as observações.

A determinação dos deslocamentos foi realizada da seguinte forma: inicialmente a plataforma foi posicionada em uma origem denominada de P0 e 
foram efetuadas as medidas no interferômetro e com as estações totais. Este ponto encontra-se mais afastado do emissor do laser, sendo que para as demais posições da plataforma foi deslocado de uma determinada distância, aproximando a plataforma do canhão emissor do sinal laser.

Como mencionado, posteriormente a plataforma foi movida para outras posições e novamente foram efetuadas as mesmas medidas realizadas na origem. Com estas observações foram determinados e comparados os deslocamentos. Na figura 2 é apresentado de forma esquemática o posicionamento da plataforma.

Figura 2 - Representação esquemática da determinação do deslocamento.

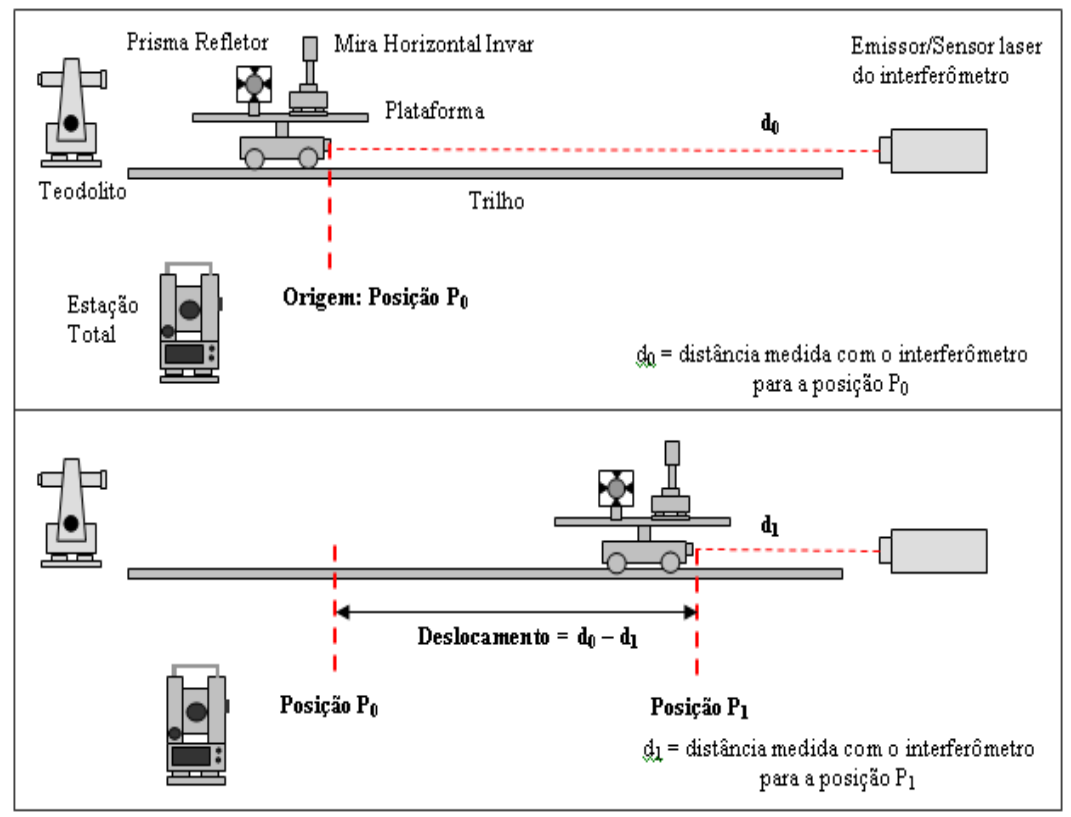

2.1 Determinação dos deslocamentos empregando-se o Interferômetro Laser.

Para determinar o deslocamento entre os pontos com precisão e servir de parâmetro para comparação com os resultados obtidos com estações totais, foi empregado o sistema de interferometria laser. De acordo com FAGGION (1993) o interferômetro permite a determinação de deslocamentos nas condições em que está instalado no LaIG, com precisão na ordem do centésimo do milímetro.

A posição inicial da plataforma onde estão instalados o refletor, a mira invar e o refletor móvel do interferômetro foi tomada como sendo igual a leitura $0,000 \mathrm{~mm}$ no interferômetro. Deslocou-se então esta plataforma e foi realizada a medição deste deslocamento com o sistema interferométrico. Os valores obtidos serão depois comparados com os medidos nas demais técnicas.

Bol. Ciênc. Geod., sec. Artigos, Curitiba, v. 19, no 1, p.34-44, jan-mar, 2013. 


\subsection{Determinação do deslocamento utilizando a Estação Total}

Para a determinação dos deslocamentos utilizando as estações totais selecionadas instalou-se um prisma refletor posicionado sobre a plataforma móvel. Desta forma, o deslocamento foi obtido a partir da resolução de um triângulo, cujos vértices são a estação total (ponto denominado de E) e o prisma, em duas posições diferentes (figura 3). A estação foi instalada sobre um tripé industrial de forma a garantir a estabilidade da mesma durante as observações.

Figura 3 - Esquema da medição com a estação total.

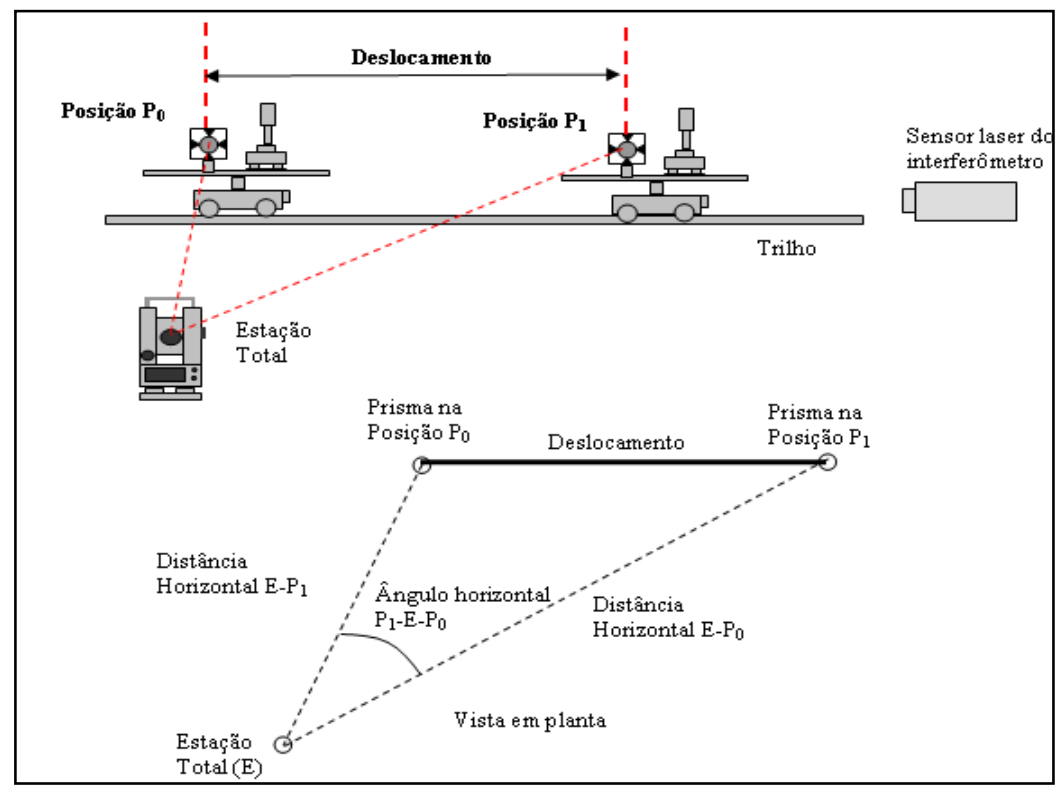

A partir do triângulo formado pela estação total e a posição do refletor $\mathrm{P}_{0}$ e $\mathrm{P}_{1}$ é possível através da lei dos cossenos, determinar a distância entre estes pontos (equação 1).

$$
\text { Deslocamento }=\sqrt{\mathrm{EP}_{1}^{2}+\mathrm{EP}_{0}^{2}-\left(2 \cdot \mathrm{EP}_{1} \cdot \mathrm{EP}_{0} \cdot \cos \left(\widehat{P_{1} E P_{0}}\right)\right)}
$$

Foram efetuadas leituras em pontaria direta e invertida da luneta para os pontos definidos pelos prismas refletores em cada uma das posições medidas. Não foram efetuadas as correções dos efeitos meteorológicos das distâncias, considerando que as duas distância a serem medidas para a determinação do deslocamento apresentavam diferenças inferiores a $60 \mathrm{~cm}$ e durante o experimento, a temperatura interna do laboratório foi mantida constante. 
Foram definidas 6 posições de parada da plataforma (definindo cinco distâncias de referência), conforme apresenta a Tabela 2. Nesta tabela são apresentadas as distâncias em relação a origem de medida de interferômetro para cada uma das posições e os respectivos deslocamentos calculados. O deslocamento da plataforma para atingir as posições intermediárias sobre os trilhos foi feito de forma manual e o controle das distâncias através do display de medida do interferômetro. As medidas foram realizadas com a plataforma se movendo do ponto mais afastado $\left(\mathrm{P}_{\mathrm{o}}\right)$ para a origem do sistema de medição e no sentido contrário.

Tabela 2 - Posições de leitura medidas com o interferômetro.

\begin{tabular}{c|c|c|c}
\hline $\begin{array}{c}\text { Posição } \\
\text { de } \\
\text { Leitura }\end{array}$ & $\begin{array}{c}\text { Posições (leituras) } \\
\text { medidas com } \\
\text { Interferômetro }(\mathrm{mm})\end{array}$ & \multicolumn{2}{|c}{$\begin{array}{c}\text { Deslocamentos de } \\
\text { referência } \\
(\mathrm{mm})\end{array}$} \\
\hline P0 & 4212,16 & $\mathrm{D}_{\mathrm{P} 0 \mathrm{P} 1}$ & 400,15 \\
\hline P1 & 3812,01 & $\mathrm{D}_{\mathrm{P} 1 \mathrm{P} 2}$ & 1000,01 \\
\hline P2 & 2812,00 & $\mathrm{D}_{\mathrm{P} 2 \mathrm{P} 3}$ & 1500,00 \\
\hline P3 & 1312,00 & $\mathrm{D}_{\mathrm{P} 3 \mathrm{P} 4}$ & 500,00 \\
\hline P4 & 812,00 & $\mathrm{D}_{\mathrm{P} 4 \mathrm{P} 5}$ & 811,99 \\
\hline P5 & 0,01 & & \\
\hline
\end{tabular}

\subsection{Propagação de variâncias.}

Para poder ter uma estimativa da precisão do deslocamento determinado empregando-se as estações totais foi utilizado o conceito de propagação de variâncias. De acordo com CHRZANOWSKI (1977), seja F uma função de várias medidas independentes $\mathrm{m}_{1}, \mathrm{~m}_{2}, \ldots, \mathrm{m}_{\mathrm{k}}$ e assumindo que o desvio padrão $\sigma_{\mathrm{m}}$ das medidas são conhecidos e que estas estão livres de erros grosseiros e sistemáticos. Neste caso é possível calcular o desvio padrão $\sigma_{\mathrm{F}}$ da quantidade $\mathrm{F}$ a partir da lei de propagação de erros:

$$
\sigma_{\mathrm{F}}^{2}=\left(\frac{\partial \mathrm{F}}{\partial \mathrm{m}_{1}}\right)^{2} \sigma_{\mathrm{m}_{1}}^{2}+\left(\frac{\partial \mathrm{F}}{\partial \mathrm{m}_{2}}\right)^{2} \sigma_{\mathrm{m}_{2}}^{2}+\ldots+\left(\frac{\partial \mathrm{F}}{\partial \mathrm{m}_{\mathrm{k}}}\right)^{2} \sigma_{\mathrm{m}_{\mathrm{k}}}^{2}
$$

Onde $\partial \mathrm{F} / \partial \mathrm{m}$ são as derivadas parciais e seus valores podem ser calculados com derivadas da função $\mathrm{F}$ para valores aproximados do correspondente levantamento. Esta equação representa a lei especial de propagação das variâncias. A lei geral de propagação das variâncias para equações lineares e não lineares é dada por (WOLF, GHILANI, 1997):

Onde:

$$
\Sigma_{\mathrm{zz}}=\mathrm{A} \Sigma \mathrm{A}^{\mathrm{T}}
$$

$\Sigma_{z z}$ é matriz de covariância para a função $Z$;

$\Sigma$ é a matriz covariância para as medidas; 
A é a matriz Jacobiana (derivada parciais).

No presente estudo o modelo matemático que correlaciona a grandeza a ser calculada (deslocamento relativo) e as observações é a lei dos cossenos. Como observações têm-se duas distâncias e um ângulo.

O ângulo é obtido a partir da leitura de pares conjugados em cada uma das posições de parada, sendo que a diferença entre as duas direções observadas permite a obtenção do ângulo. A precisão deste ângulo será função da precisão nominal angular da estação total. Normalmente para uma estação total, a precisão nominal angular é definida para um par de leituras conjugadas de uma direção. Assim, para uma estação total de 5" ao se efetuar a leitura da direção horizontal em um alvo em pontaria direta e depois em pontaria invertida, este valor indica que o desvio padrão da média das duas direções será de 5" (Professional Surveyor Magazine, 2002). Para um ângulo (diferença entre a leitura de duas direções efetuadas em pontaria direta e invertida) a precisão será de 7 segundos $\left(5^{\prime \prime} \sqrt{2}\right)$.

Para o cálculo da precisão da distância medida será utilizada a equação descrita em WOLF,GUILANE (1997), que permite estimar o erro de uma distância medida com um MDE (medidor eletrônico de distância):

$$
\sigma_{\mathrm{D}}=\sqrt{\sigma_{\mathrm{i}}^{2}+\sigma_{\mathrm{t}}^{2}+\mathrm{a}^{2}+(\mathrm{D} \cdot \mathrm{b} p \mathrm{ppm})^{2}}
$$

Onde:

$\sigma_{\mathrm{D}}$ é o erro na distância medida;

$\sigma_{\mathrm{i}}$ é o erro de centragem do instrumento;

$\sigma_{t}$ é o erro de centragem do refletor;

a é parte constante da precisão de um MED;

b é parte variável da precisão de um MED;

D é a distância medida.

Neste experimento os valores de $\sigma_{i}$ e $\sigma_{t}$ serão considerados iguais a zero, visto o equipamento estar instalado em um tripé industrial de centragem forçada e o prisma estar fixo na plataforma móvel do interferômetro. Na Tabela 3 são apresentados os resultados da propagação para duas situações, a primeira para o deslocamento $\mathrm{D}_{\mathrm{P} 0 \mathrm{P} 1}$ (distâncias aproximadas de $3,95 \mathrm{~m}$ e $4,0 \mathrm{~m}$, com um ângulo de 5 graus) e $\mathrm{D}_{\mathrm{P} 4 \mathrm{P} 5}$ (distâncias aproximadas de $4,8 \mathrm{~m}$ e $5,3 \mathrm{~m}$, com um ângulo de 7 graus).

Tabela 3 - Precisões estimadas para os deslocamentos.

\begin{tabular}{c|c|c}
\hline $\begin{array}{c}\text { Estação Total } \\
\text { Precisão linear / angular }\end{array}$ & $\begin{array}{c}\mathrm{D}_{\mathrm{P} 0 \mathrm{P} 1} \\
(\mathrm{~mm})\end{array}$ & $\begin{array}{c}\mathrm{D}_{\mathrm{P} 4 \mathrm{P} 5} \\
(\mathrm{~mm})\end{array}$ \\
\hline TC2003 $\pm(1 \mathrm{~mm}+1 \mathrm{ppm}) / \pm 1 ”$ & 0,2 & 0,8 \\
\hline Elta S20 $\pm(2 \mathrm{~mm}+2 \mathrm{ppm}) / \pm 3 "$ & 0,4 & 1.8 \\
\hline TC407 $\pm(5 \mathrm{~mm}+2 \mathrm{ppm}) / \pm 7 ”$ & 1,1 & 4,4 \\
\hline GTS239 $\pm(3 \mathrm{~mm}+3 \mathrm{ppm}) / \pm 9 "$ & 0,7 & 2,7 \\
\hline
\end{tabular}




\section{RESULTADOS}

A Tabela 4 apresenta as leituras efetuadas com a estação total TC2003 para as posições P5 e P4, exemplificando as observações realizadas. São apresentadas as leituras das direções horizontais, ângulos zenitais e distâncias inclinadas medidas em pontaria direta e inversa para as posições indicadas.

Tabela 4 - Observações com a estação total TC2003 para as posições P5 e P4

\begin{tabular}{c|c|c|c}
\hline - & Pontaria Direta & Pontaria Inversa & Posição de leitura \\
\hline Direção Horizontal & $358^{\circ} 45^{\prime} 37,3^{\prime \prime}$ & $178^{\circ} 45^{\prime} 35,2^{\prime \prime}$ & P4 \\
\hline Ângulo Zenital & $89^{\circ} 24^{\prime} 58,1^{\prime \prime}$ & $270^{\circ} 34^{\prime} 51^{\prime \prime}$ & $812,00 \mathrm{~mm}$ \\
\hline Distância inclinada & $4,8367 \mathrm{~m}$ & $4,8367 \mathrm{~m}$ & \\
\hline Direção Horizontal & $05^{\circ} 52^{\prime} 37,1^{\prime \prime}$ & $185^{\circ} 52^{\prime} 38^{\prime \prime}$ & P5 \\
\hline Ângulo Zenital & $89^{\circ} 28^{\prime} 28,6^{\prime \prime}$ & $270^{\circ} 31^{\prime} 19,9^{\prime \prime}$ & $0,01 \mathrm{~mm}$ \\
\hline Distância inclinada & $5,3474 \mathrm{~m}$ & $5,3474 \mathrm{~m}$ & - \\
\hline
\end{tabular}

A Tabela 5 apresenta os resultados dos deslocamentos relativos determinados para cada uma das estações totais. De posse das observações, aplicou-se o modelo matemático apresentado (equação 1) para a determinação dos deslocamentos entre cada posição. Os resultados são apresentados na Tabela 5.

Tabela 5 - Deslocamentos calculados.

\begin{tabular}{c|c|c|c|c|c}
\hline- & \multicolumn{5}{|c}{ Deslocamentos $(\mathrm{mm})$} \\
\hline Estação Total & $\mathrm{D}_{\mathrm{P} 4 \mathrm{P} 5}$ & $\mathrm{D}_{\mathrm{P} 3 \mathrm{P} 4}$ & $\mathrm{D}_{\mathrm{P} 2 \mathrm{P} 3}$ & $\mathrm{D}_{\mathrm{P} 1 \mathrm{P} 2}$ & $\mathrm{D}_{\mathrm{POP} 1}$ \\
\hline TC2003 - IDA & 811,97 & 499,82 & 1499,95 & 1000,19 & 400,18 \\
\hline TC2003 - VOLTA & 811,99 & 499,89 & 1499,95 & 999,84 & 400,59 \\
\hline Elta S20 - IDA & 811,77 & 499,56 & 1499,45 & 999,24 & 399,99 \\
\hline Elta S20 - VOLTA & 811,66 & 499,82 & 1498,86 & 999,66 & 400,34 \\
\hline TC407 - IDA & 812,12 & 499,53 & 1500,01 & 999,65 & 400,06 \\
\hline TC407 - VOLTA & 812,16 & 499,33 & 1500,29 & 999,47 & 400,10 \\
\hline GTS239- IDA & 808,20 & 502,28 & 1500,84 & 1000,55 & 400,86 \\
\hline GTS239 - VOLTA & 811,50 & 498,96 & 1501,70 & 999,59 & 401,29 \\
\hline
\end{tabular}

A Tabela 6 apresenta as diferenças entre os deslocamentos determinados com as Estações Totais e Interferômetro. 
Tabela 6 - Diferenças entre os deslocamentos determinados com as Estações Totais e Interferômetro.

\begin{tabular}{c|c|c|c|c|c}
\hline- & \multicolumn{7}{|c}{ Diferenças $(\mathrm{mm})$} \\
\hline Equipamento & $\mathrm{D}_{\mathrm{P} 4 \mathrm{P} 5}$ & $\mathrm{D}_{\mathrm{P} 3 \mathrm{P} 4}$ & $\mathrm{D}_{\mathrm{P} 2 \mathrm{P} 3}$ & $\mathrm{D}_{\mathrm{P} 1 \mathrm{P} 2}$ & $\mathrm{D}_{\mathrm{P} 0 \mathrm{P} 1}$ \\
\hline TC2003 - IDA & 0,0 & $-0,2$ & $-0,1$ & 0,2 & 0,0 \\
\hline TC2003 - VOLTA & 0,0 & $-0,1$ & 0,0 & $-0,2$ & 0,4 \\
\hline Elta S20 - IDA & $-0,2$ & $-0,4$ & $-0,6$ & $-0,8$ & $-0,2$ \\
\hline Elta S20 - VOLTA & $-0,3$ & $-0,2$ & $-1,1$ & $-0,3$ & 0,2 \\
\hline TC407 - IDA & 0,1 & $-0,5$ & 0,0 & $-0,4$ & $-0,1$ \\
\hline TC407 - VOLTA & 0,2 & $-0,7$ & 0,3 & $-0,5$ & 0,0 \\
\hline GTS239 - IDA & $-3,9$ & 2,7 & 0,8 & 0,9 & 0,8 \\
\hline GTS239 - VOLTA & $-0,5$ & $-1,0$ & 1,7 & $-0,4$ & 1,1 \\
\hline
\end{tabular}

Foram calculadas médias das diferenças encontradas, bem como a média absoluta (desconsiderando o sinal da diferença). Os resultados são apresentados na Tabela 7.

Tabela 7 - Médias das diferenças.

\begin{tabular}{c|c|c}
\hline Equipamento & $\begin{array}{c}\text { Média das } \\
\text { diferenças }(\mathrm{mm})\end{array}$ & $\begin{array}{c}\text { Média das diferenças } \\
\text { absolutas }(\mathrm{mm})\end{array}$ \\
\hline TC2003 & 0,0 & 0,1 \\
\hline Elta S20 & $-0,4$ & 0,4 \\
\hline TC407 & $-0,2$ & 0,3 \\
\hline GTS239 & 0,2 & 1,4 \\
\hline
\end{tabular}

Para a estação GTS $239 \mathrm{~W}$, durante a realização da leitura na posição P4 no sentido de ida, pode ter ocorrido algum problema na pontaria, o que acabou afetando o resultado dos deslocamentos $\mathrm{D}_{\mathrm{P} 4 \mathrm{P} 5}$ e $\mathrm{D}_{\mathrm{P} 3 \mathrm{P} 4}$. Desconsiderando estes dois valores, a média das diferenças resulta em $0,4 \mathrm{~mm}$ e a média absoluta em $0,9 \mathrm{~mm}$.

\section{CONCLUSÕES}

Os resultados obtidos neste estudo de caso mostram que, para o experimento realizado em laboratório, as diferenças encontradas nos valores dos deslocamentos determinados com as estações totais, quando comparados com os valores padrão, ficaram abaixo do milímetro. Contribuíram para este resultado o fato do experimento ser realizado em ambiente com temperatura controlada, a instalação do equipamento feita em um tripé industrial que garante a estabilidade do mesmo para aplicações indoor e as distâncias entre o equipamento de medida e os prismas serem pequenas (inferiores a $5 \mathrm{~m}$ ). Deve-se ressaltar também que os resultados foram obtidos com a realização de apenas uma série de leituras conjugadas nos prismas, sendo que o aumento do número de séries tende a melhorar os resultados, visto 
aumentar o número de observações para cada uma das posições. Desta forma os resultados mostram que, para as condições do experimento, foi possível determinar os deslocamentos com precisão sub-milimétrica, e melhor que a estimada através dos processos matemáticos de propagação da variância para a configuração de medida empregada. Recomenda-se a realização de novos experimentos com distâncias maiores e em ambientes não controlados.

\section{BIBLIOGRAFIA CONSULTADA}

CHRZANOWSKI, A. Design and error analysis of surveying projects. University of New Brunswick, 1977.

FAGGION, P. L. Contribuição para a implantação de um sistema de aferição de miras de ínvar na UFPR. Curitiba, 1993, Dissertação (Curso de PósGraduação em Ciências Graduação em Ciências Geodésicas), Setor de Ciência da Terra, Universidade Federal do Paraná.

PROFESSIONAL SURVEYOR MAGAZINE. DIN 18723 Specification for theodolite Accuracy. Professional Surveyor Magazine, November, 2002.

WOLF, P.R.; GHILANI, C. D. Adjustment Computations: statistics and least squares in surveying and GIS. 3 ed. New York. Editora: John Wiley \& Sons. 1997.

(Recebido em setembro de 2012. Aceito em dezembro de 2012.) 